\title{
Optimal mathematical and statistical models to estimate vertical crustal movements using satellite altimetry and tide gauge data
}

DOI: https://doi.org/10.1515/jogs-2019-0014

Received May 28, 2019; accepted November 26, 2019

\begin{abstract}
Knowledge of vertical crustal movement is fundamental to quantify absolute sea level changes at tide gauge locations as well as for satellite altimetry calibration validations. While GPS measurements at collocated tide gauge stations fulfill this need, currently only few hundred tide gauge stations are equipped with GPS, and their measurements do not span a long period of time. In the past, several studies addressed this problem by calculating relative and geocentric trends from the tide gauge and satellite altimetry measurements respectively, and then difference the two trends to calculate the rate of changes at the tide gauge stations. However, this approach is suboptimal. This study offers an optimal statistical protocol based on the method of condition equations with unknown parameters. An example solution demonstrates the proposed mathematical and statistical models' optimality in estimating vertical crustal movement and its standard error by comparing them with the results of current methods. The proposed model accounts for the effect of autocorrelations in observed tide gauge and satellite altimetry sea level time series, adjusts observed corrections such as inverted barometer effects, and constraints tide gauge and satellite altimeter measurement to close. The new model can accommodate estimating other systematic effects such as pole tides that are not eliminated by differencing.
\end{abstract}

\footnotetext{
*Corresponding Author: H. Bâki Iz: Division of Geodetic Science, School of Earth Sciences, The Ohio State University, Columbus, Ohio, USA, E-mail: h.baki.iz@gmail.com

T. Y. Yang: Division of Geodetic Science, School of Earth Sciences, The Ohio State University, Columbus, Ohio, USA

C. K. Shum: Division of Geodetic Science, School of Earth Sciences, The Ohio State University, Columbus, Ohio, USA and State Key Laboratory of Geodesy and Earth's Dynamics, Institute of Geodesy \& Geophysics, Chinese Academy of Sciences, Wuhan, Hubei 430077, China

C. Y. Kuo: Department of Geomatics, National Cheng Kung University, Tainan, Taiwan., T. Y. Yang, C. K. Shum, C. Y. Kuo
}

Keywords: Vertical crustal movements; Satellite altimetry; Tide gauges; Sea level rise; Optimal statistical solution; Condition equations with unknown parameters

\section{Introduction}

Knowledge of vertical motion is fundamental to quantify absolute sea level changes at tide gauge (TG) locations, and for satellite altimetry (SA) calibration validations. While global positioning system (GPS) measurements at collocated TG stations also fulfill this need, currently only few hundred TG stations are equipped with GPS and span a sufficiently long period of time. Several studies, such as Cazenave et al. (1999), Nerem and Mitchum (2002), Kuo et al. (2004), Garcı'a et al. (2007), Ray et al., (2010), and others addressed this problem by differencing the estimated rate of change in sea level time series observed by both SA (geocentric rates) and TG (relative rates) to the extent the sea level signals are sufficiently similar over the same area. The difference of the estimated linear trend is a measure of vertical crustal movement (VCM) experienced at a TG station.

Nonetheless, the recovery of the VCM is by the current studies is sub-optimal ${ }^{1}$ due to the omission of autocorrelations in TG and SA time series, which leads to Type I errors in testing null-hypotheses about the estimated parameters. Although studies by Bouin and Wöppelmann (2010), Ray et al. (2010) recognized the existence of the autocorrelations in TG and SA time series and accounted for in error bars of the estimated VCM, their approach is also suboptimal as compared to the proposed protocol deployed in this study. All these studies are also sub-optimal because they apply corrections rather than adjust corrections that are also observed quantities, such as Inverted Barometer (IB) effects, or corrections for tidal variations and others.

1 An optimal, as opposed to a sub-optimal estimation gives the lower bounds of the quadratic risk, such as mean-square error, scalar mean square error, for heterogeneous and homogeneous linear set-ups. See Chapter 2.4 of Toutenburg (1982) for further details. 
More importantly none of these studies has imposed conditions in the estimation of the VCM to ensure that the misclosures of the TG - SA differences referring to the same time tags are adjusted. This condition is necessary to calculate external variability of the model variables holistically.

The proposed mathematical and statistical models in this study address all these deficiencies while adjusting all the pertinent observed time series simultaneously. Consequently, proposed approach gives the optimal estimates for the VCM at a TG station and its standard error and all the other solution statistics. The mathematical and statistical models are demonstrated using an example solution at the Key West, USA TG station in the following sections. The estimated VCM at the Key West TG station is then compared to the results obtained using existing models in recent literature.

\section{Key West Tide Gauge and Satellite Altimetry Data}

The Key West TG station was selected for the numerical demonstration of the proposed statistical model. TG time series were downloaded from the Permanent Mean Sea Level (PSMSL) repository (PSMSL, 2018, Holgate et al., 2013) and truncated to include only overlapping time period 1993 - 2017 with the available SA time series during the same time span (Fig. 1). TG time series are referenced to the Revised Local Reference (RLR). Because no corrections for the post glacial rebound (PGR) or local vertical movements were applied to the data, they represent relative sea level changes with respect to the Earth's crust.

The sea level around the Key West TG station was also surveyed by various satellite altimetry for geocentric mean sea level (MSL) change. We used sea level anomaly data produced by the NASA's MEaSURe's program, which were downloaded on January 2019 for the region $24^{0} \mathrm{~N}, 82^{0} \mathrm{~W}-25^{\circ} \mathrm{N}, 81^{0} \mathrm{~W}$ for the period Dec 1992 - Jun 2017 (Fig. 1). The time series includes data from TOPEX/Poseidon (T/P), Jason-1, Ocean Surface Topography Mission/Jason-2 (OSTM) and Jason-3 primary mission series (TPJAOS) (Zlotnicki et al., 2016).

\subsection{Inverted Barometer Effect at Key West Tide Gauge Station}

Although the SA measurements are already corrected for the effect of atmospheric pressure TG measurements are

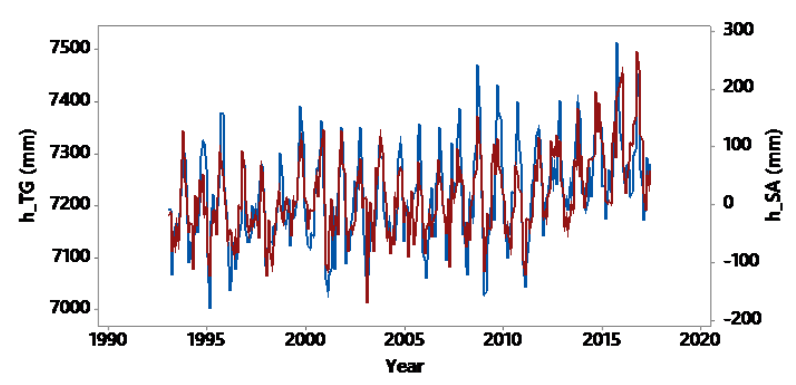

Fig. 1. Overlapping TG (in blue) and SA monthly sea level measurements in $\mathrm{mm}$. Each time series refers to its own datum.

not. In this study, ERA-Interim monthly averaged surface pressure product was used for computing inverted barometer (IB) correction (Dee et al., 2011). The ERA-Interim dataset for the Key West TG station was downloaded from European Centre for Medium-Range Weather Forecasts archive (ECMWF, 2019, Berrisford et al., 2011). The data reflect the monthly averaged surface pressure on a regular $0.75^{\circ} \times 0.75^{\circ}$ longitude/latitude grid for the period from Jan 1979 to Oct 2018 (Fig. 2). Before computing IB correction, surface pressure data in land area is removed in whole dataset.

The relation between surface pressure and IB correction is given by the following equation (Wunsch, 1997),

$$
I B_{(\varphi, \lambda)}^{t}=\frac{-1}{\rho g}\left(P_{(\varphi, \lambda)}^{t}-\bar{P}_{(\varphi, \lambda)}^{t}\right)=-9.948\left(P_{(\varphi, \lambda)}^{t}-\bar{P}_{(\varphi, \lambda)}^{t}\right)
$$

where

$$
\bar{P}_{(\varphi, \lambda)}^{t}=\frac{\sum_{\varphi=90^{\circ}}^{-90^{\circ}}\left[\cos (\varphi) \times \sum_{\lambda=0^{\circ}}^{360^{\circ}} P_{(\varphi, \lambda)}^{t}\right]}{\sum_{\varphi=90^{\circ}}^{-90^{\circ}}\left[\cos (\varphi) \times n_{\varphi}\right]}
$$

In these expressions, $I B_{(\varphi, \lambda)}^{t}$ is $I B$ correction at time epoch $t,(\varphi, \lambda)$ are the latitude and longitude of the TG station, $\rho$ is ocean density, $g$ is gravity acceleration, $P_{(\varphi, \lambda)}^{t}$ is the monthly averaged surface pressure, $\bar{P}_{(\varphi, \lambda)}^{t}$ which is spatially averaged pressure over the global surface via Eq. (2), $n_{\varphi}$ is the number of non-zero $P_{(\varphi, \lambda)}^{t}$ at each latitude. In Eq. (1), $1 / \rho g(\mathrm{~mm} / \mathrm{mb})$ is a scale factor based on empirical value at mid-latitude. The $I B$ corrections for the Key West TG station are displayed on Fig. 2. The data span 1993-2017 overlaps with the TG and SA data. 


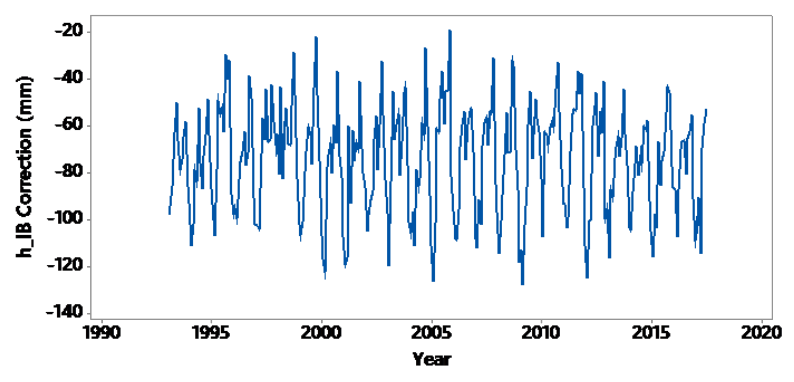

Fig. 2. IB correction at Key West TG station.

\subsection{Vertical movement estimation using trend differencing with ordinary least squares}

The following model is used to estimate the linear rate of change in sea level rise $\dot{h}$ for both TG and SA time series,

$$
\begin{aligned}
h_{t}= & h_{t_{0}}+\dot{h}\left(t-t_{0}\right)+\alpha \cos \left(\frac{2 \pi}{P_{\text {Annual }}}\right)\left(t-t_{0}\right) \\
& +y \sin \left(\frac{2 \pi}{P_{\text {Annual }}}\right)\left(t-t_{0}\right)+u_{t}
\end{aligned}
$$

It is assumed that the disturbances, $u_{t}$, has the following distributional properties,

$$
\begin{array}{r}
E\left(u_{t}\right)=0 \quad E\left(u_{t}^{2}\right)=\sigma_{u}^{2} \quad E\left(u_{t} u_{t-1}\right)=0 \\
\left(t \neq t^{\prime}\right) \rightarrow \Sigma_{u}=\operatorname{diagonal}\left(\sigma_{u_{t}}^{2}\right)
\end{array}
$$

In this representation, monthly $\mathrm{TG}$ or SA observations at an epoch $t$ is denoted by $h_{t}$. The time index $t=1 \ldots n$, where $n$ is the number of months and includes all the available data. The intercept $h_{t_{0}}$ is the datum offset of either TG or SA series referenced to the epoch $t_{0}$ chosen to be in the middle of the series. Trend, i.e., the linear rate of change in sea level, experienced by the series is denoted by $\dot{h}$, which is identified as relative sea level trend, which is affected by the VCM at the TG station and the geocentric sea level trend of SA, which independent of the VCM. The cyclic annual variations are modeled with two unknown parameters, $\alpha_{h}, y_{h}$ for the sine and cosine components from which the amplitudes $a_{h}$ and the phase angles of the periodic terms are determined. In total, the model includes four unknown parameters, which are solved using the ordinary least squares (OLS). The difference of the estimated rates of SA and TG time series gives the linear rate of change in VCM, $\hat{\dot{h}}^{V C M}$ at the TG station,

$$
\hat{\dot{h}}^{V C M}=\hat{\dot{h}}_{t}^{S A}-\hat{\dot{h}}_{t}^{T G_{C}}
$$

In this expression, the estimated SA sea level trend denoted by $\hat{h}_{t}^{S A}$ and $\hat{h}_{t}^{T G_{C}}$, where circumflex denotes an estimated parameter, refers to the estimated relative sea level trend using TG measurements corrected a priori for the IB effect.

The estimated trends for the Key West SA and TG series for this model using OLS are $4.27 \pm 0.39$ and $4.53 \pm 0.55 \mathrm{~mm} / \mathrm{yr}$ respectively. Given the use of the two different technologies, the errors of SA and TG measurements are assumed to be statistically independent. Hence, the error of the estimated VCM is obtained using variance propagation,

$$
\hat{\sigma}_{\hat{h}^{V C M}}^{2}=\hat{\sigma}_{\hat{h}_{t}^{S A}}^{2}+\hat{\sigma}_{\hat{h}_{t}^{T G} C}^{2}
$$

The estimated rate of trend of VCM and its uncertainty at the Key West TG station using OLS is then $-0.26 \pm 0.67 \mathrm{~mm}$ /yr. (Table 1.).

Note that the estimated SA and TG sea level rates are both biased because of the omission of the low frequency variations in sea level, which are not estimable in short SA series. Nonetheless, differencing eliminates these effects because SA and TG measurements both sense the same sea level variations, hence the estimated trend biases are the same. Meanwhile, the plots of the residuals for both series in Fig. 3 reveal non-linearities that are not explained by the models.

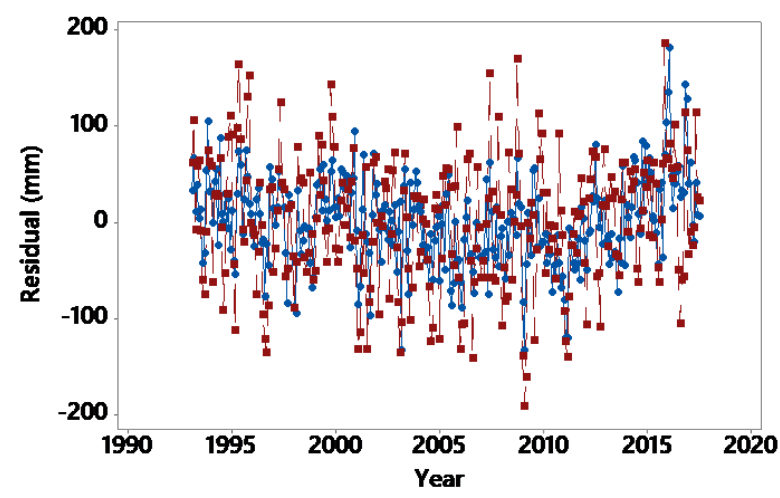

Fig. 3. Residuals of the SA (Blue) and TG solutions.

Figure 4 exhibits the correlograms of the residuals of both series and reveals statistically significant first order autocorrelations, $\operatorname{AR}(1)$. The magnitudes of the AR(1) correlation coefficients are approximately the same, $\rho \simeq 0.5$. The autocorrelations, if not modeled, will not impact the trend estimates but will cause a Type I error (i.e. failing to reject the null-hypothesis) in testing the statistical significance of the estimated VCM because of the overly optimistic biased standard errors of the estimates (Neter et al., 1997). The remedy for this problem is the topic for the following section. 
Table 1. All velocities are in mm/yr at Key West TG station. GIA velocities were calculated using the ensemble average of $13 \mathrm{GIA}$ models (Iz et al., 2017g) at the location: (24.560, -81.810). NA: Not Applicable.

\begin{tabular}{|c|c|c|c|c|c|c|c|}
\hline $\begin{array}{l}\text { GPS Station } \\
\text { (Obs. Period) }\end{array}$ & $\begin{array}{l}\text { Distance } \\
\text { (km) }\end{array}$ & $\hat{i}^{G P S}$ & $\hat{h}^{G / A}$ & $\begin{array}{l}\hat{h}^{S A}-\hat{h}^{T G} \\
\mathrm{w} / \mathrm{o} A R(1)\end{array}$ & $\begin{array}{c}\hat{b}^{S A}-\hat{v}^{T G} \\
\operatorname{AR}(1)\end{array}$ & $\begin{array}{c}\hat{h}^{S A-T G} \\
\mathrm{w} / \mathrm{o} A R(1)\end{array}$ & $\begin{array}{l}\hat{h}^{S A-T G} \\
\operatorname{AR}(1)\end{array}$ \\
\hline $\begin{array}{c}\text { KWST } \\
(2002-2004) \\
\text { KYW1 }\end{array}$ & 5 & $-1.07 \pm 0.38$ & $-0.73 \pm 0.08$ & $-0.26 \pm 0.67$ & $-0.33 \pm 1.20$ & $-0.26 \pm 0.34$ & $-0.26 \pm 0.24$ \\
\hline $\begin{array}{c}(1996-2007) \\
\text { KYW5 }\end{array}$ & 16 & $0.26 \pm 0.43$ & $-0.73 \pm 0.08$ & $-0.26 \pm 0.67$ & $-0.33 \pm 1.20$ & $-0.26 \pm 0.34$ & $-0.26 \pm 0.24$ \\
\hline $\begin{array}{c}\text { (2007-2016) } \\
\text { Weighted }\end{array}$ & 15 & $1.76 \pm 0.43$ & $-0.73 \pm 0.08$ & $-0.26 \pm 0.67$ & $-0.33 \pm 1.20$ & $-0.26 \pm 0.34$ & $-0.26 \pm 0.24$ \\
\hline Mean & NA & $1.03 \pm 0.24$ & $-0.73 \pm 0.08$ & $-0.26 \pm 0.67$ & $-0.33 \pm 1.20$ & $-0.26 \pm 0.34$ & $-0.26 \pm 0.24$ \\
\hline
\end{tabular}
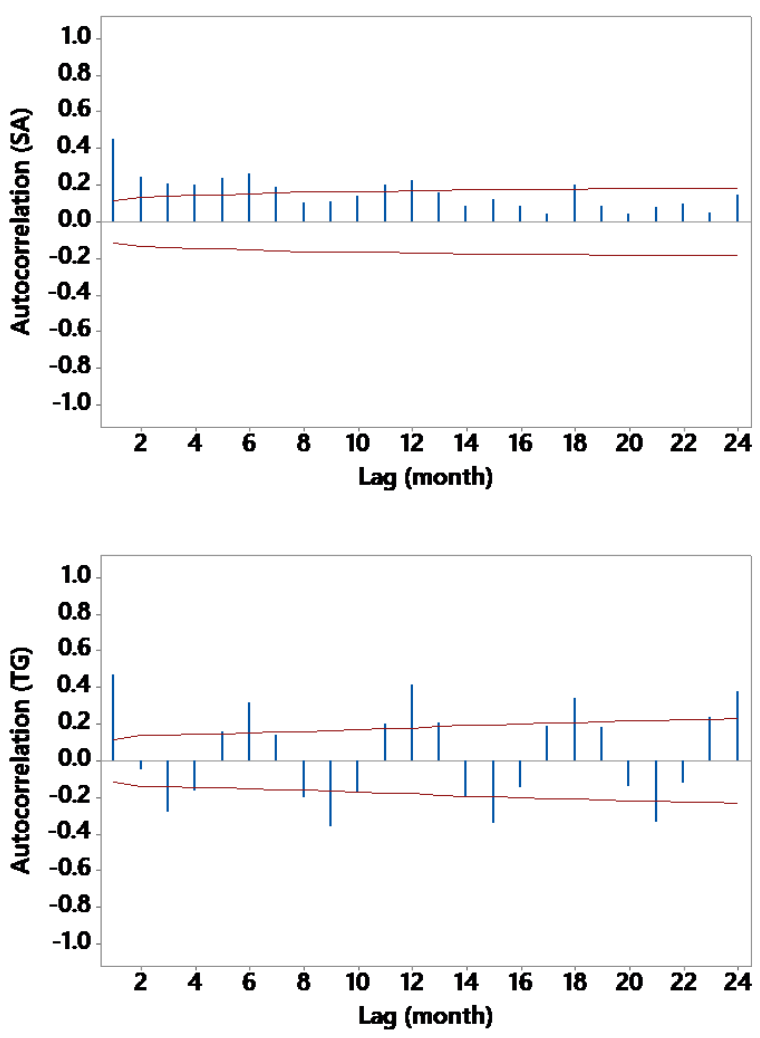

Fig. 4. Correlelograms of the SA and TG residual series. Confidence intervals (95\%) are in red.

\subsection{Statistical trend models with autoregressive disturbances}

As demonstrated in the previous section, and repeatedly reported by Iz et al. (2012m - 2018a) sea level measurements, irrespective of their origins, exhibit first order positive autocorrelation AR(1) for various reasons. Autocorrelation (serial correlation) in measurements arise from observation errors in the included variables, from the estimation of missing data by either averaging or extrapolating.
Even unmodeled periodic sea level variations induce autocorrelations. In sea level studies, positive first order autocorrelation coefficients of the time series are of varying degree as a function of location of the measurements and the time interval between averaged measurements. The autocorrelations are not confined to TG observations but also present in SA time series for the sea level anomalies.

If these correlations are not accounted for in the estimation process, the resulting variances of the estimated parameters are overestimated. Hence, autocorrelations induce Type I errors, which lead to falsely claiming an estimated model parameter is statistically significant. This problem will lead to catastrophic conclusions, like the presence of a sea level acceleration when there is none in the presence of unmodeled positive first order autocorrelations. To ameliorate this problem a first order autoregressive, also known as linear Markov process, is incorporated into the estimation as part of the proposed statistical protocol. (Tautenburg, 1982, İz and Chen, 1999).

The following derivation is given by Tautenburg (1982). The vector of disturbances for a time series is assumed to be autocorrelated. A first order autoregressive process $\mathrm{AR}(1)$ for each element of disturbances is expressed as,

$$
e_{t}=\rho e_{t-1}+u_{t} \quad t=\ldots,-2,-1,0,1,2, \ldots
$$

where $-1 \leq \rho \leq 1$ is the unknown autocorrelation coefficient. The stochastic process, $\left\{u_{t}\right\}$, has the following assumed distributional properties,

$$
\begin{array}{r}
E\left(u_{t}\right)=0 \quad E\left(u_{t}^{2}\right)=\sigma_{e}^{2} \quad E\left(u_{t} u_{t-1}\right)=0 \\
\left(t \neq t^{\prime}\right) \rightarrow \Sigma_{u}=\operatorname{diagonal}\left(\sigma_{u_{t}}^{2}\right)
\end{array}
$$

An equivalent expression for Eq. (7) is therefore,

$$
e_{t}=\sum_{s=0}^{\infty} \rho^{s} u_{t-s}
$$

Considering Eq. (8) and the properties of the geometric series, it can be shown that,

$$
E\left(e_{t}\right)=0, \quad \operatorname{var}\left(e_{t}\right)=E\left(e_{t}^{2}\right)=\left(1-\rho^{2}\right)^{-1} \sigma_{u_{t}}^{2}=\sigma^{2}
$$


For a sample size $n$, the $\mathrm{V} / \mathrm{C}$ matrix of the disturbances for any one of the autocorrelated time series, after some manipulations, can be written as,

$$
\Sigma=\sigma^{2} \cdot\left[\begin{array}{ccccc}
1 & \rho & \rho^{2} & \cdots & \rho^{n-1} \\
\rho & 1 & \cdots & \cdots & \rho^{n-2} \\
\vdots & \vdots & \vdots & \ddots & \vdots \\
\rho^{n-1} & \rho^{n-2} & \rho^{n-3} & \cdots & 1
\end{array}\right]=\sigma^{2} \cdot \mathbf{P}^{-1}
$$

Implicit in this result is the assumption that measurements are equally spaced in time. The correlation decreases for increasing time lag because $|\rho|<1$. The above patterned $\mathrm{V} / \mathrm{C}$ matrix has an analytical inverse, and is given by,

$$
\begin{aligned}
\Sigma^{-1} & =\frac{\sigma^{-2}}{1-\rho^{2}} \mathbf{P} \\
& =\left[\begin{array}{cccccc}
1 & -\rho & 0 & \cdots & 0 & 0 \\
-\rho & 1+\rho^{2} & -\rho & \cdots & 0 & 0 \\
0 & -\rho & 1+\rho^{2} & \cdots & 0 & 0 \\
\vdots & \vdots & \vdots & \ddots & \vdots & \vdots \\
0 & 0 & 0 & \cdots & 1+\rho^{2} & -\rho \\
0 & 0 & 0 & \cdots & -\rho & 1
\end{array}\right]
\end{aligned}
$$

From Eq. (11), the correlation between two autocorrelated random variables $e_{t}$ and $e_{t-\tau}$ is $\sigma^{2} \rho^{\tau}$, where $\tau$ is the time lag.

With this a priori understanding regarding the autocorrelated error properties of the SA and TG time series, the trend model given by Eq. (3) can now be expressed as,

$$
\begin{aligned}
h_{t}= & h_{t_{0}}+\dot{h}\left(t-t_{0}\right)+\alpha \cos \left(\frac{2 \pi}{P_{\text {Anпиа }}}\right)\left(t-t_{0}\right) \\
& +y \sin \left(\frac{2 \pi}{P_{\text {Anпиа }}}\right)\left(t-t_{0}\right)+e_{t}
\end{aligned}
$$

where the disturbances, $e_{t}$, are autocorrelated.

This model was used to calculate the trends for the $\mathrm{SA}$ and TG time series using least squares with $\mathrm{AR}(1)$ errors. The estimated trends are $4.33 \pm 0.70 \mathrm{~mm} / \mathrm{yr}$ and $4.66 \pm 0.98 \mathrm{~mm} / \mathrm{yr}$ for the Sa and TG time series respectively (Table 1). The new estimates are not markedly different than those obtained using OLS, which are 4.27 \pm 0.39 and $4.53 \pm 0.55 \mathrm{~mm} / \mathrm{yr}$. for the $\mathrm{SA}$ and TG series respectively. Nonetheless, their standard errors are doubled in size because of the effect of the autocorrelations. The difference of the two estimates that gives the estimate for the rate of VCM at the TG station is $-0.33 \pm 1.20 \mathrm{~mm} / \mathrm{yr}$. This estimate is similar to the estimated VCM rate of $-0.26 \pm 0.67 \mathrm{~mm} / \mathrm{yr}$. obtained using OLS, but its standard error is twice as large as it should be.

Modelling the inclusion of the AR(1) ameliorated the problem of underestimated standard errors of the VCM rate by ignoring it. Yet, this is still not the best approach to estimate the rate of VCM because of the way the IB effect is corrected. So far, the IB correction assumed that atmospherics pressure measurements are errorless. This assumption is incorrect. Moreover, the correlogram for the IB series (Fig. 5) reveals that the IB series are also autocorrelated with a statistically significant AR(1) correlation coefficient $\rho_{I B}=0.6$ at $95 \%$ confidence level. Adjusting for the effect of autocorrelations in IB series in estimating VCM to get realistic estimates for the rates and their standard errors of VCM at TG stations is therefore necessary. This is topic of the next section.

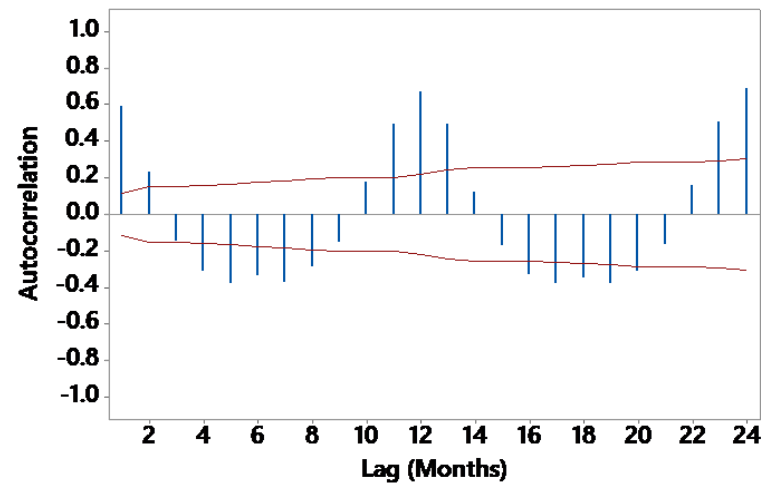

Fig. 5. Correllogram for the IB series. AR(1) correlation coefficient is 0.6. Confidence intervals (95\%) are in red.

\section{Proposed mathematical and statistical models}

A typical TG measurement requires corrections for the effect of atmospheric pressure, VCM experienced by the station, high and low frequency tides, etc. In the following model, we will consider only the inverted barometer effect, IB, for corrections and the effect of the VCM. This practice is not because all the other effects are negligible, but as discussed before, differencing eliminates errors common to SA and TG time series ${ }^{2}$. The emphasis here is to account for the effect of the autocorrelations in IB series as mea-

2 There were also residual yearly sea level variations that are not canceled by differencing of the TG and SA time series. The amplitude of this residual effect was statistically significant but did not impact estimated VCM rates significantly, hence not included in this example for simplicity in the presentation. 
surements subject to errors in estimating rate of VCM at a TG station.

An IB and VCM corrected TG measurement at a given epoch $t$ subject to an unknown VCM is given by,

$$
h_{t}^{T G_{C}}=h_{t}^{T G}+h_{t}^{I B}+h_{t}^{V C M}
$$

In this expression $h_{t}^{T G_{C}}, h_{t}^{T G}, h_{t}^{I B}$ and $h_{t}^{V C M}$ refer to the corrected TG measurement, observed TG record, IB effect, and the unknown VCM correction at the TG station at an epoch $t$ respectively. If we denote $h_{t}^{S A}$ as a nearby sea level height as observed by SA at the same epoch $t$ of TG measurement, then each of the IB corrected TG measurement and SA measurement is expected to close, i.e. to fulfil the following condition,

$$
h_{t}^{T G_{C}}-h_{t}^{S A}=0
$$

Inserting Eq. (15) in Eq. (16), we obtain,

$$
h_{t}^{T G}+h_{t}^{I B}+h_{t}^{V C M}-h_{t}^{S A}=0
$$

If VCM at an epoch $t$ at a TG station has a linear trend, $\dot{h}^{V C M}$, then,

$$
h_{t}^{V C M}=\delta^{V C M}+\dot{h}^{V C M}\left(t-t_{0}\right)
$$

where $\delta^{V C M}$ is the reference geocentric height of the TG station at an epoch $t_{0}$.

In the above equations, all the observed time series are subject to random measurement errors, which can be modeled as,

$$
\begin{gathered}
h_{t}^{T G}=\delta^{T G}+h_{t}^{T G_{O B S}}+e_{t}^{T G_{O B S}} \\
h_{t}^{I B}=\delta^{I B}+h_{t}^{I B_{O B S}}+e_{t}^{I B_{O B S}} \\
h_{t}^{S A}=\delta^{S A}+h_{t}^{S A_{O B S}}+e_{t}^{S A_{O B S}}
\end{gathered}
$$

where $\delta^{T G}, \delta^{I B}$ and $\delta^{S A}$ are the unknown offsets of TG measurements from a datum, a constant offset of the IB corrections, and a reference offset of SA measurements.

Substituting Eqn. (18) through Eqn. (21) in Eqn. (17), we obtain the following condition equation with unknown parameters,

$$
e_{t}^{T G_{O B S}}+e_{t}^{I B_{O B S}}-e_{t}^{S A_{O B S}}+\delta+\dot{h}^{V C M}\left(t-t_{0}\right)+w=0
$$

where,

$$
\delta:=\delta^{T G_{O B S}}+\delta^{I B_{O B S}}-\delta^{S A_{O B S}}
$$

is the lumped offsets ${ }^{3}$. The misclosure, $w$, is given by,

$$
w:=h_{t}^{V C M}+h_{t}^{T G_{O B S}}+h_{t}^{I B_{O B S}}-h_{t}^{S A_{O B S}}
$$

where $\delta$ and $v^{V C M}$ are the unknown parameters to be estimated. In calculating the misclosure equation it is assumed the unknown VCM velocity is zero. If prior information is available about its size, it can also be included in the misclosure with the provision that the unknown parameter for the VCM velocity would be a correction to the prior information about it. In this case, the condition equations with unknown parameters will reduce to the method of condition equations only. Such a formulation is itself an ideal way for the conflation of GPS derived VCMs with TGSA derived VCMs.

In this formulation, the $\mathrm{V} / \mathrm{C}$ matrices and their inverses for the error terms of TG, SA and IB time series can be modeled using the derivations in the previous section. The first order autoregressive correlation coefficients $\rho_{T G}, \rho_{S A}, \rho_{I B}$ are already estimated from the residuals of their series after their trends and their systematic variations are removed. Also, TG, IB and SA measurements are not correlated because they were carried out independently. Therefore, the combined V/C matrix for all the time series is given by,

$$
\underset{3 n \times 3 n}{\Sigma^{-1}}=\left[\begin{array}{ccc}
\sigma_{e_{T G}}^{-2} \mathbf{P}_{T G} & 0 & 0 \\
0 \times n & \sigma_{n \times n} & 0 \\
0 & \sigma_{e_{I B}}^{-2} \mathbf{P}_{I B} & 0 \\
\underset{n \times n}{n \times n} & \underset{n \times n}{0} & \sigma_{e_{S A}}^{-2} \mathbf{P}_{S A} \\
0 \times n & 0
\end{array}\right]
$$

Note that the elements of the above $\mathrm{V} / \mathrm{C}$ matrix should be rearranged to conform with eq. (26), which are not shown here. The formulation for the solution for the condition equations with unknown parameters given by Eq. (19), Eq. (20), and Eq. (21) together with AR(1) disturbances including those from the IB series is the topic of the following section.

\section{Condition equations with unknown parameters}

The method of condition equation with unknown parameters was formulated by Friedrich Robert Helmert (18431917), a geodesist. In this section, we do not include its derivation for brevity but followed the outcome of the derivations and notations used in Uotila (1988) with minor modifications and simplifications.

Now, we are ready to express the linear mathematical model stated in the earlier section that contains observations as well as unknown parameters in matrix notation for $n$ observations in each series with $r$ conditions equa-

3 “:=” reads as "defined as" 
tions and $m$ unknown parameters as follows ${ }^{4}$,

$$
\underset{r \times n}{\mathbf{B}} \underset{n \times 1}{\mathbf{V}}+\underset{r \times m}{\mathbf{A}} \underset{m \times 1}{\mathbf{X}}+\underset{r \times 1}{\mathbf{W}}=\underset{r \times 1}{\mathbf{0}}
$$

The weight matrix associated with the observations is denoted by $\underset{n \times n}{\mathbf{P}}$. The principle of the minimum variance solution requires minimizing the $\mathbf{V}^{\mathrm{T}} \mathbf{P V}$ and fulfilling the conditions imposed on the observations can be obtained using the method of Lagrange multipliers for the following target function:

$$
\mathbf{V}^{\mathbf{T}} \mathbf{P V}-2 \lambda^{\mathbf{T}}(\mathbf{B V}+\mathbf{A X}+\mathbf{W})=\text { stationary }
$$

Where $\lambda$ is an $r \times 1$ vector of Lagrange multipliers. The solution includes the following compendium of equations (ibid).

The unknown parameters are calculated using the following expression:

$$
\hat{\mathbf{X}}=-\left(\mathbf{A}^{\mathrm{T}} \mathbf{M}^{-\mathbf{1}} \mathbf{A}\right)^{-\mathbf{1}} \mathbf{A}^{\mathrm{T}} \mathbf{M}^{-1} \mathbf{W}
$$

with the corresponding variance-covariance, V/C matrix,

$$
\Sigma_{\hat{\mathbf{X}}}=\hat{\sigma}_{0}^{2}\left(\mathbf{A}^{\mathbf{T}} \mathbf{M}^{-\mathbf{1}} \mathbf{A}\right)^{-\mathbf{1}}
$$

where $\mathbf{M}:=\mathbf{B P}^{-\mathbf{1}} \mathbf{B}^{\mathbf{T}}$. The a posteriori variance of unit weight $\hat{\sigma}_{0}^{2}$ is given by,

$$
\hat{\sigma}_{0}^{2}=\frac{\mathbf{V}^{\mathbf{T}} \mathbf{P V}}{r-m}
$$

The number of unknown VCM parameters is this example is 2; the unknown lumped datum offsets, and the VCM velocity. The proposed model can now be quantified using all three the time series to estimate VCM at the Key West TG station.

\section{Adjustment of TG and SA misclosures at the Key West TG station and estimation of the VCM}

The proposed mathematical and statistical model was used to estimate vertical crustal movements at the Key West TG station using TG, IB and SA time series. Monthly misclosures calculated using Eq. (24) are shown in Fig. 6.

\footnotetext{
4 Note that this is a generic derivation. Dimensions of the matrices should be quantified following the mathematical model discussed in the previous section. Notation for the disturbances are replaced by their estimates, i.e. "residuals" and the weight matrix $\mathrm{P}$ is introduced in relation to the inverse $\mathrm{V} / \mathrm{C}$ matrix of disturbances with a unit weight of one to preserve the original notation used in the derivation given by Uotila (1997).
}

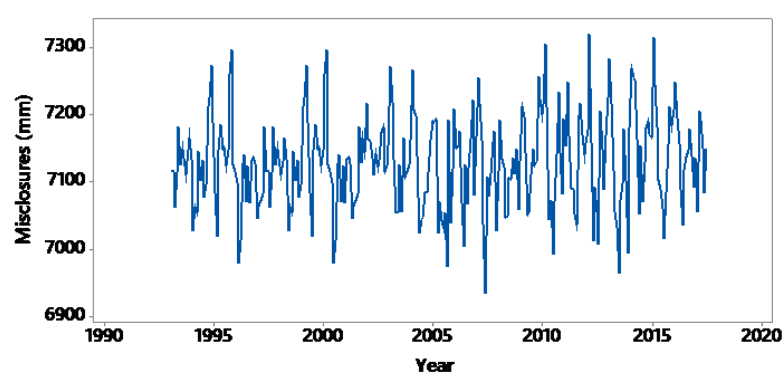

Fig. 6. $\mathrm{RMSE}=68.8 \mathrm{~mm}$.

The estimated rate of VCM experienced by the TG station using this approach is $-0.26 \pm 0.24 \mathrm{~mm} / \mathrm{yr}$., which indicates that the station may not have experienced a statistically significant VCM during this period. The magnitude of the VCM is similar to those estimated using trend differences, with and without autoregressive disturbances but the standard error of the VCM rate is the smallest among the alternatives (Table 1). The adjusted residuals for the TG, IB and SA time series are displayed on Fig. 7, Fig. 8, and Fig. 9. All the residuals do not pass Anderson-Darling normality test because of the high variation but do not show any unmodeled systematic variation.

Another run without modelling the $\mathrm{AR}(1)$ reveal that the results are not markedly different, $-0.26 \pm 0.24$ vs. $0.26 \pm 0.34 \mathrm{~mm} / \mathrm{yr}$. The robustness of the estimated parameters can be attributed to differencing the monthly time series instead of their trends, which are demonstrably affected by the autocorrelated disturbances. Differencing of the monthly observations disturbs the systematic effect of autocorrelations and enables similar results for solutions with or without autocorrelated disturbances.

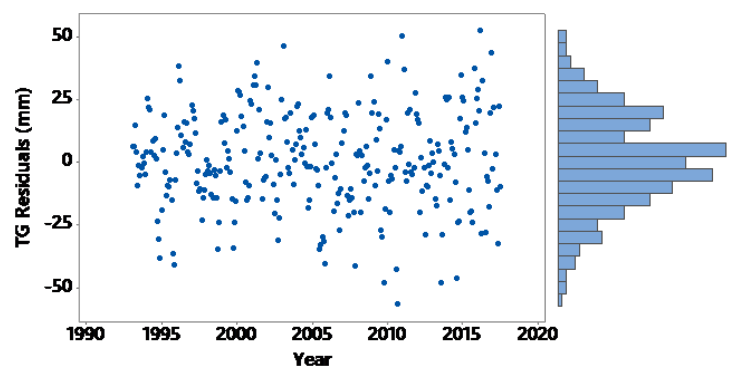

Fig. 7. TG residuals are in $\mathrm{mm}$. RMSE of the residuals is $19.2 \mathrm{~mm}$. 


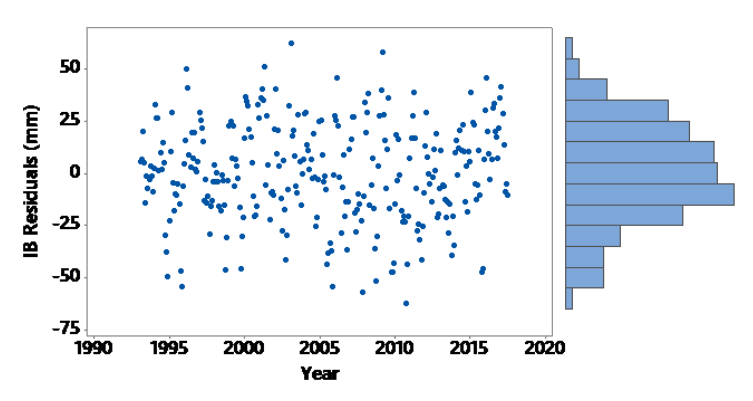

Fig. 8. IB residuals in $\mathrm{mm}$. RMSE of the residuals is $23.5 \mathrm{~mm}$.

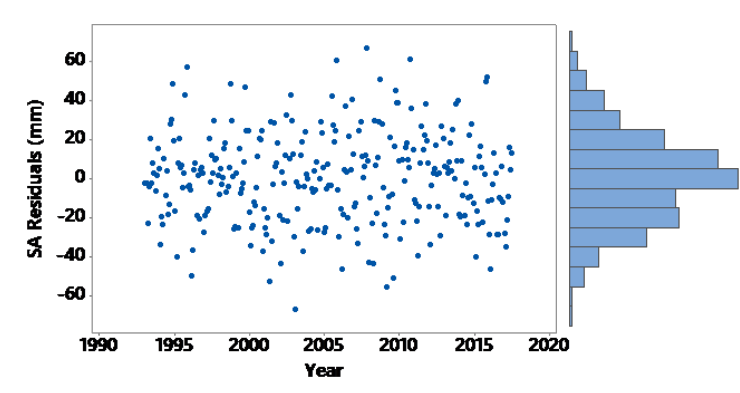

Fig. 9. SA residuals are in $\mathrm{mm}$. RMS of the residuals is $23.1 \mathrm{~mm}$.

\section{Verification: Vertical crustal velocities by GPS, GIA, and TG-SA measurements}

In this section, the estimated linear rates of VCM from different model solutions are contrasted with the linear VCM rates calculated using GPS measurements and GIA induced trend for the surrounding region. The estimated VCM rates derived from GPS measurements were downloaded from SONEL ULR6a GPS solution, which is a version of the reanalysis of 19 years of GPS data from 1995 to 2014 (Santamaría-Gómez et al., 2017). The GIA velocity is calculated using the ensemble average of 13 GIA models (Iz et al., 2017g) at the location $\left(24.56^{\circ},-81.81^{\circ}\right)$.

First entries in Table 1 are the VCM rates using GPS measurements carried out at nearby locations to the Key West TG station. The GPS stations are nearly located with the Key West TG station, yet there are no links, such as precise levelling that tie the GPS stations to the TG station to evaluate the contribution of local subsidence/uplifts at the GPS stations and the TG station. Although the weighted mean of the VCM rates of GPS origin is statistically significant at $5 \%$ significant level, the individual GPS based VCM rates at nearby stations are inconsistent. These results are indicative of similar limitations that will be experienced at other TG stations. The marked variability of the esti- mated GPS velocities at three clustered around the nearby TG stations affirms our initial assertion that the short span GPS time series are insufficient to resolve VCMs with confidence. Yet GPS is still the best mean for quantifying VCMs in the long run.

As an alternative verification, GIA velocities were calculated using the ensemble average of $13 \mathrm{GIA}$ models (Iz et al., 2017g) at the location: $\left(24.56^{\circ},-81.81^{\circ}\right)$. Despite the ensemble average is statistically significant and exhibit subsidence like the VCM estimated at the TG station, the average is only a rough approximation for the VCM rate because it does not account for the local VCM at the Key West TG station.

Because the local contribution, such as site settlement and hydrological loading to the VCM experienced at the TG station is unknown, the estimated VCMs for verification are biased. Therefore, the null hypothesis " $\mathrm{H}_{0}: T G$ station does not experience any statistically significant VCM" cannot be rejected due to the large standard error of various estimates in conjunction with the small magnitude of the VCM rate.

The IB corrections applied to the SA measurements may also be another source of large standard errors of the estimated VCM rates using different models. Downloaded SA time series were already corrected using model based IB corrections and SA series are observationally smoother than the observed atmospheric pressure variations converted into IB corrections. A better practice for future studies is therefore not to apply any IB corrections for both TG and SA time series. Because both series experience the same effect, differencing will eliminate the IB effects naturally. These limitations however are common for all alternative model solutions in practice, hence have no impact in assessing the optimality of the proposed approach.

The ensemble average of the VCM induced by GIA for this area is precise because the GIA models use the same data in model building hence their ensemble averages tend to agree with each other. Meanwhile, the estimated VCM rate for this exercise is small, and again, the absence of information about the local subsidence/uplift is prohibitive for allowing a reliable comparison with the GIA predicted VCM rate and other estimates. Yet, comparison of the estimated VCM rates with the ensemble average predicted by the 13 GIA models is still informative. All estimated VCM rates using SA and TG series indicated VCM as a subsidence as in the case of predicted GIA rate. 


\section{Conclusion}

This study offered an optimal statistical protocol for estimating VCM at TG stations using TG, SA and IB time series data using the method of condition equations with unknown parameters. The solution accounts for the effect of autocorrelations in observed TG and SA sea level time series, adjusts the observed correction, the IB effect and constraints TG and satellite altimeter measurement to close, while estimating VCM at the TG station. The solution is demonstrated to be efficient compared to the estimates using alternative models using SA, TG and IB time series related to the Key West TG as an example. Given the short time span of the GPS measurements collocated or located nearly to most TG stations around the globe, the use of TG and SA measurements in conjunction, as demonstrated in this study, is an effective alternative to estimate VCM at globally distributed thousands of TG stations. The statistical protocol is statistically optimal in the sense that it yields estimates consistent with their alternatives, it does so with significantly smaller standard errors with clear improvements over both GPS and alternative sub-optimal satellite altimetry/tide-gauge solutions.

Acknowledgments: We thank to an anonymous reviewer for comments that greatly improved the manuscript.

\section{References}

Bjerhammer, A., 1973, Theory of errors and generalized matrix inverses, Elsevier Scientific Publishing Co., Amsterdam.

Beckley, B., Zelensky, N.P., Holmes, S.A., Lemoine, F.G., Ray, R.D., Mitchum, G.T., Desai, S., Brown, S.T., 2017, Integrated Multi-Mission Ocean Altimeter Data for Climate Research complete time series Version 4.2. Ver. 4.2. PO.DAAC, CA, USA. Dataset accessed [2019-01-03] at http://dx.doi.org/10.5067/ALTTS-TJ142.

Bouin, M. N., and G. Wöppelmann, 2010, Land motion estimates from GPS at tide gauges: a geophysical evaluation Geophys. J. Int., Vol. 180, pp.193-209.

Dee, D.P., Uppala, S.M., Simmons, A.J., Berrisford, P., Poli, P., Kobayashi, S., Andrae, U., Balmaseda, M.A., Balsamo, G., Bauer, D.P. and Bechtold, P., 2011, The ERA-Interim reanalysis: Configuration and performance of the data assimilation system. Quarterly Journal of the royal meteorological society, 137(656), pp.553-597.

Gabriele G., J. Feichter, Eds., 2011, Climate change and policy: The calculability of climate change and the challenge of uncertainty, Springer, 241 pgs.

García, D., Vigo, I., Chao, B. et al., 2007, Pure Appl. Geophys. 164: 851.
Helmert, F. R.,1872, Ausgleichungsrechnung nach der Methode der kleinsten Quadrate, mit Anwendungen auf die Geodäsie und die Theorie der Messinstrumente.

Hildreth, C. and Lu, J.Y., 1960, Demand relationships with autocorrelated disturbances. Michigan State University. Agric. Exp. Statn. Bulletin, 276.

Holgate, S.J., Matthews, A., Woodworth, P.L., Rickards, L.J., Tamisiea, M.E., Bradshaw, E., Foden, P.R., Gordon, K.M., Jevrejeva, S. and Pugh, J., 2013, New data systems and products at the permanent service for mean sea level. Journal of Coastal Research, 29(3), pp.493-504.

İz H.B., C.K. Shum, C.Y. Kuo, 2018a, Sea Level Accelerations at Globally Distributed Tide Gauge Stations During the Satellite Altimetry Era, J. Geod. Sci. Vol. 8, pp. 130-135.

İ H.B., 2018b, Why and How to Predict Sea Level Changes at a Tide Gauge Station with Prediction Intervals, J. Geod. Sci. Vol. 8, pp. 121-129.

İ H.B., 2018c, Is the global sea surface temperature rise accelerating? Geodesy and Geodynamics, 1-7 (in press). https://doi.org/10.1016/j.geog.2018.04.002

İz H.B., 2018d, The Effect of Regional Sea Level Atmospheric Pressure to Sea Level Variations at Globally Distributed Tide Gauge Stations with Long Records, J. Geod. Sci. Vol. 8, pp. 55-71.

İz H.B., C.K. Shum, 2018e, Regional Absolute Sea Level Rise from a Cluster of Tide Gauge Stations. Preprint: DOI 10.13140/RG.2.2.11625.60005.

İz H.B., 2017f, Acceleration of the Global Coastal Sea Level Rise During the 20th Century Re-evaluated, J. Geod. Sci., Vol. 7, pp. 51-58.

İz H.B., C.K. Shum, C. Zhang, C.Y. Kuo, 2017g, Inferring Regional Vertical Crustal Velocities from Averaged Relative Sea Level Velocities, J. Geod. Sci., Vol. 7, pp. 59-67.

İz H.B., 2016h, Thermosteric Contribution of Warming Oceans to the Global Sea Level Variations, J. Geod. Sci., Vol. 6, pp. 130-138.

İ H.B., 2016i, The Effect of Warming Oceans at a Tide Gauge Station, J. Geod. Sci., Vol. 6, pp. 69-79.

İ H.B., 2015j, More Confounders at Global and Decadal Scales in Detecting Recent Sea Level Accelerations, J. Geod. Sci., Vol. 5, pp. 192-198.

Iz H.B., 2014k, Sub and Super Harmonics of the Lunar Nodal Tides and the Solar Radiative Forcing in Global Sea Level Changes, J. Geod. Sci., Vol. 4, pp. 150-165.

İz H.B., X.L. Ding, and C.K. Shum, 2013l, Global Sea Level Trends in the Presence of Variable Sea Level Velocities, and Variable Accelerations, J. Geod. Sci., Vol. 3, Issue 2, pp. 127-135.

İz H.B., L. Berry, and M. Koch, 2012m, Modeling regional sea level rise using local tide gauge data, J. Geod. Sci., Vol. 2, Issue 3, pp. 188-1999.

Iz H.B. and Y. Chen, 1999, VLBI Rates with First Order Autoregressive Disturbances, Journal of Geo-dynamics, Vol. 28, No. 2-3, pp. 131-145

Kuo, C.Y., Shum, C.K., Braun, A. and Mitrovica, J.X., 2004, Vertical crustal motion determined by satellite altimetry and tide gauge data in Fennoscandia. Geophysical Research Letters, 31(1).

Mayer, T., in Stigler, S. M., 1986, The History of Statistics: The Measurement of Uncertainty before 1900. Cambridge, Mass.: Belknap Press of Harvard University Press.

Nerem, R.S. and Mitchum, G.T., 2002, Estimates of vertical crustal motion derived from differences of TOPEX/POSEIDON and tide 
gauge sea level measurements. Geophysical Research Letters, 29(19), pp.40-1.

Ray, R.D., Beckley, B.D. and Lemoine, F.G., 2010, Vertical crustal motion derived from satellite altimetry and tide gauges, and comparisons with DORIS measurements. Advances in Space Research, 45(12), pp.1510-1522.

Santamaría-Gómez, A., Gravelle, M., Dangendorf, S., Marcos, M., Spada, G. and Wöppelmann, G., 2017, Uncertainty of the 20th century sea-level rise due to vertical land motion errors. Earth and Planetary Science Letters, 473, pp.24-32.

Permanent Service for Mean Sea Level (PSMSL), 2018, Tide Gauge Data, Retrieved 24 Oct 2018 from http://www.psmsl.org/data/obtaining/.

Toutenburg, H., 1982, Prior information in linear models, John Wile and Sons, New York.
Uotila, U., 1988, Analysis of Observations. Lecture Notes, The Ohio State University.

WCRP Global Sea Level Budget Group: Global sea-level budget 1993-present, 2018, Earth Syst. Sci. Data, 10, 1551-1590, https://doi.org/10.5194/essd-10-1551-2018.

Wunsch, C. and Stammer, D., 1997, Atmospheric loading and the oceanic "inverted barometer" effect. Reviews of Geophysics, 35(1), pp.79-107.

Zlotnicki, Victor; Qu, Zheng; Willis, Joshua. 2016, JPL MEaSUREs Gridded Sea Surface Height Anomalies Version 1609. Ver. 1609. PO.DAAC, CA, USA. at http://dx.doi.org/10.5067/SLREFCDRV1 and Integrated Multi-Mission Ocean Altimeter Data for Climate Research TOPEX/Poseidon, Jason-1, 2, \& 3 User's Handbook Version 4.2., 2019, https://sealevel.nasa.gov/data/tools. Dataset accessed [201901]. 